\title{
Tigeciclina. Una revisión sistemática de la experiencia clínica en sus primeros años de uso
}

\author{
Alexis Diomedi P.
}

Hospital Del Salvador. Santiago, Chile. Unidad de Infectología Instituto Nacional de Oncología y Clínica Los Coihues, Santiago.

Recibido: 13 de junio 2008 Aceptado: 6 agosto 2008

\section{Correspondencia a:} Alexis Diomedi Pacheco adiomedip@gmail.com adiomedip@terra.cl

\section{Tigecycline: A systematic review of clinical experience during first years of prescription}

The available clinical experience with tigecycline is analyzed under the perspective of a systematic review of the literature, so in the already approved indications as in those off label indications reported in the recent literature. The safety profile is checked in the above mentioned clinical trials. The available information allows supporting tigecycline efficiency in the managing of complicated skin and soft tissues infections, complicated intrabdominales infections and community acquired pneumonias. Its usefulness is insinuated in addition in the managing infection by pathogen with high-level of resistance to antimicrobial. Nevertheless it is needed of major evidence in the matter and of a very sensible policy of use in the healthcare institution setting.

Key words: Tigecycline, clinical trials, efficacy, effectiveness, post marketing.

Palabras clave: Tigeciclina, estudios ensayos, eficacia, efectividad, post licencia.
$\mathrm{T}$ igeciclina es el primer representante licenciado de las glicilciclinas, análogos de las tetraciclinas descubiertos en 1993, con la particular propiedad de superar los principales mecanismos de resistencia bacteriana de sus antecesores, fundamentalmente relacionados con mecanismos de eflujo y protección ribosomal ${ }^{1}$. Dado el potencial de su uso en infecciones por microorganismos multi-resistentes es de capital importancia el poder sopesar su real eficacia, tanto en los estudios clínicos que llevaron a su licenciamiento como en los ensayos publicados posteriormente a tal fecha.

El objetivo de este artículo, formulado como una revisión sistemática de la literatura médica, es revisar la experiencia clínica abierta y comparativa, en las indicaciones ya aprobadas por las entidades reguladoras y en aquellas indicaciones off label reportadas en la literatura reciente. Además repasaremos su perfil de seguridad y tolerabilidad en dichos ensayos clínicos.

\section{Estrategia de búsqueda}

Se analizaron todas las referencias citadas en MEDLINE y LILACS desde el 1 de enero de 2003 al 31 de enero de 2008, considerando el término clave Tigecycline versus los demarcadores clinical trial, randomized controlled trial, case reports y letter.
Sólo se revisaron trabajos hechos en adultos a partir de los 18 años de edad. Se incluyó además la información de los resúmenes de conferencias como la Interscience Conference on Antimicrobial Agents and Chemotherapy (ICAAC), el Meeting of Infectious Diseases Society of America (IDSA) y el European Congress of Clinical Microbiology and Infectious Diseases (ECCMID), desde 2006 a la fecha. Finalmente, se obtuvo reseña adicional de Internet de revistas con edición electrónica adelantada y de otros documentos de acceso libre.

\section{Resumen de estudios clínicos}

Tigeciclina se ha evaluado, tanto en el tratamiento de infecciones de piel y tejidos blandos, como de infecciones intra-abdominales complicadas y en un estudio fase $2^{2}$ y en cuatro estudios clínicos de fase $3^{3-6}$. El resultado de estos estudios se resume en las Tablas 1 y 2 , respectivamente. Recientemente se ha comunicado el resultado de dos estudios de uso de tigeciclina en neumonía adquirida en la comunidad (Tabla 3) 7.8; $^{7}$ sin embargo, esta indicación aún no se encuentra aprobada. Actualmente, otros ensayos clínicos en curso evalúan la eficacia del antimicrobiano en neumonías adquiridas en la comunidad, neumonía nosocomial (versus imipenem), infecciones de piel y tejidos blandos de adquisición ambulatoria (versus ampicilina/ 
sulbactam o amoxicilina/ác. clavulánico), infecciones intra-abdominales complicadas (versus ceftriaxona más metronidazol) y en infecciones causadas por patógenos multi-resistentes 9 .

A continuación nos referiremos a los ensayos clínicos controlados para indicaciones específicas y luego a los reportes de uso clínico en series clínicas o reportes de casos aislados.

\section{Infecciones complicadas de piel y tejidos blandos} (ICPTB)

En un primer estudio de tipo prospectivo, abierto, multicéntrico, fase $2^{2}$, se enrolaron 169 adultos hospitalizados con infecciones de piel y tejidos blandos complicadas (edad media 49 años) entre septiembre de 1999 y marzo de 2001, siendo randomizados en proporción 1:1 para recibir una dosis de carga de $100 \mathrm{mg}$ de

\begin{tabular}{|c|c|c|c|c|c|}
\hline Estudio & Diseño & $\begin{array}{c}n \text { de } \\
\text { pacientes }\end{array}$ & Regímenes & Tasas de curación & $\begin{array}{l}\text { Efectos adversos más frecuentemente } \\
\text { reportados }(>5 \%)\end{array}$ \\
\hline \multirow[t]{2}{*}{ Postier y cols (2) } & \multirow[t]{2}{*}{ Fase $2, M C, A L, A B$} & 54 & $\begin{array}{l}\text { TGC dosis de carga } \\
100 \mathrm{mg} \text { iv y luego } \\
50 \mathrm{mg} \mathrm{c} / 12 \mathrm{hrs}\end{array}$ & $\begin{array}{l}74 \% \quad(95 \% \quad I C ; \\
60,3-85,0 . P: N S)\end{array}$ & $\begin{array}{l}\text { Náuseas }(34,6 \%) \text {, vómitos }(18,5 \%) \text {, diarrea }(8,6 \%) \text {, } \\
\text { fiebre, insomnio y dolor (todos estos } 6,2 \% \text { ) }\end{array}$ \\
\hline & & 55 & $\begin{array}{l}\text { TGC dosis de carga } \\
50 \mathrm{mg} \text { iv y luego } \\
25 \mathrm{mg} \mathrm{c} / 12 \mathrm{hrs}\end{array}$ & $\begin{array}{l}67 \%(95 \% \text { IC; } \\
53,3 \text {-79,3. P: NS) }\end{array}$ & $\begin{array}{l}\text { Náuseas }(21,5 \%) \text {, vómitos }(12,7 \%) \text {, diarrea, disnea } \\
\text { (ambos } 11,4 \%) \text {, cefalea }(12,3 \%) \text {, dolor }(8,3 \%) \text {, } \\
\text { anemia }(5,2 \%) \text {, fiebre, mareos, insomnio e hiper- } \\
\text { tensión (todos estos } 6,2 \%)\end{array}$ \\
\hline \multirow[t]{2}{*}{$\begin{array}{l}\text { Sacchidanand S } \\
\text { y cols (3) }\end{array}$} & \multirow[t]{2}{*}{ Fase 3, MC, AL, DC } & 199 & $\begin{array}{l}\text { TGC dosis de carga } \\
100 \mathrm{mg} \text { iv y luego } \\
50 \mathrm{mg} \mathrm{c} 12 \mathrm{hrs}\end{array}$ & $\begin{array}{l}82,9 \%(95 \% \mathrm{IC} \\
77,0-87,9 ; \mathrm{p}<0,001 \\
\text { para no inferioridad })\end{array}$ & $\begin{array}{l}\text { Náuseas }(43,4 \%) \text {, vómitos }(26,7 \%) \text {, diarrea } \\
(12,7 \%) \text {, cefalea }(12,3 \%) \text {, dolor }(8,2 \%) \text {, prolon- } \\
\text { gación TTPK }(6,2 \%) \text {, disnea, reacción en sitio } \\
\text { infusión iv (ambos } 5,5 \%)\end{array}$ \\
\hline & & 198 & $\begin{array}{l}\text { Vancomicina } 1 \mathrm{~g} \text { más } \\
\text { aztreonam } 2 \mathrm{~g} \text { iv } \\
\mathrm{c} / 12 \mathrm{hrs}\end{array}$ & $\begin{array}{l}82,3 \%(95 \% \text { IC; } \\
76,3-87,4)\end{array}$ & $\begin{array}{l}\text { Náuseas }(11,0 \%) \text {, prurito }(10,7 \%) \text {, cefalea }(9,3 \%) \text {, } \\
\text { diarrea }(8,5 \%) \text {, fiebre }(7,5 \%) \text {, flebitis, alza ALT } \\
\text { (ambos } 5,7 \%) \text {, constipación }(5,3 \%)\end{array}$ \\
\hline \multirow[t]{2}{*}{ Breedt y cols (4) } & \multirow[t]{2}{*}{ Fase $3, \mathrm{MC}, \mathrm{AL}, \mathrm{DC}$} & 223 & $\begin{array}{l}\text { TGC dosis de carga } \\
100 \mathrm{mg} \text { iv y luego } \\
50 \mathrm{mg} \mathrm{c} / 12 \mathrm{hrs}\end{array}$ & $\begin{array}{l}89,7 \%(95 \% I C ; \\
84,9-93,3 ; p<0,001 \\
\text { para no inferioridad })\end{array}$ & $\begin{array}{l}\text { Náuseas }(25,2 \%) \text {, trombocitosis }(15,4 \%) \text {, } \\
\text { vómitos }(12,0 \%)\end{array}$ \\
\hline & & 213 & $\begin{array}{l}\text { Vancomicina } 1 \mathrm{~g} \text { más } \\
\text { aztreonam } 2 \mathrm{~g} \text { iv } \\
\mathrm{c} / 12 \mathrm{hrs}\end{array}$ & $\begin{array}{l}94,4 \%(95 \% \text { IC; } \\
90,4-97,1)\end{array}$ & $\begin{array}{l}\text { Linfopenia }(13,9 \%) \text {, trombocitosis }(8,9 \%) \text {, alza } \\
\text { de ALT, alza de AST, náuseas, hipertensión arterial } \\
\text { (todos, } 5,2 \%)\end{array}$ \\
\hline
\end{tabular}

\section{Tabla 2. Resumen de ensayos clínicos de tigeciclina en infecciones intra-abdominales complicadas}

\begin{tabular}{|c|c|c|c|c|c|}
\hline Estudio & Diseño & $\begin{array}{c}n \text { de } \\
\text { pacientes }\end{array}$ & Regímenes & Tasas de curación & $\begin{array}{l}\text { Efectos adversos más frecuentemente } \\
\text { reportados }(>5 \%)\end{array}$ \\
\hline Oliva y cols (5) & Fase $3, \mathrm{MC}, \mathrm{AL}, \mathrm{DC}$ & 341 & $\begin{array}{l}\text { TGC dosis de carga } \\
100 \mathrm{mg} \text { iv y luego } \\
50 \mathrm{mg} \mathrm{c} / 12 \mathrm{hrs} \\
\text { Imipenem/cilastatina } \\
500 \mathrm{mg} \text { iv c/6 h (o } \\
\text { ajuste según clearance } \\
\text { de creatinina) }\end{array}$ & $\begin{array}{l}82,7 \%(95 \% \mathrm{IC} \\
78,3-86,6 ; p<0,001 \\
\text { para no inferioridad }) \\
84,0 \%(95 \% \mathrm{IC} ; \\
79,8-87,7)\end{array}$ & $\begin{array}{l}\text { Náuseas }(31,0 \%) \text {, vómitos }(25,7 \%) \text {, diarrea } \\
(21,3 \%) \text {, infección }(13,6 \%) \text { hipoproteinemia } \\
(8,0 \%) \text {, disnea }(6,8 \%) \\
\text { Náuseas ( } 24,8 \%) \text {, vómitos }(19,4 \%) \text {, diarrea } \\
(19,4 \%) \text {, infección }(7,5 \%)\end{array}$ \\
\hline Dartois y cols (6) & Fase $3, \mathrm{MC}, \mathrm{AL}, \mathrm{DC}$ & 346 & $\begin{array}{l}\text { TGC dosis de carga } \\
100 \mathrm{mg} \text { IV y luego } \\
50 \mathrm{mg} \mathrm{c} / 12 \mathrm{hrs} \\
\text { Imipenem/cilastatina } \\
500 \mathrm{mg} \text { IV c/6 h (o } \\
\text { ajuste según clearance } \\
\text { de creatinina) }\end{array}$ & $\begin{array}{l}90,7 \%(95 \% \text { IC NA } \\
p<0,001 \text { para no } \\
\text { inferioridad }) \\
90,2 \%(95 \% \text { IC NA) }\end{array}$ & $\begin{array}{l}\text { Náuseas }(17,6 \%) \text {, vómitos }(12,6 \%) \text {, reacción en } \\
\text { sitio infusión iv }(6,9 \%) \text {, infección }(6,7 \%) \text {, diarrea } \\
(6,2 \%) \text {, trombocitopenia }(6,2 \%) \\
\text { Náuseas }(13,30 \%) \text {, vómitos }(9,2 \%) \text {, diarrea }(7,5 \%) \text {, } \\
\text { fiebre }(7,3 \%) \text {, reacción en sitio infusión iv }(6,5 \%) \text {, } \\
\text { trombocitopenia }(6,1 \%)\end{array}$ \\
\hline
\end{tabular}

MC: multicéntrico; AL: aleatorio; $\mathrm{AB}$ : abierto; DC: doble ciego; TGC: tigeciclina. 
tigeciclina y luego $50 \mathrm{mg}$ cada $12 \mathrm{hr}$, o una dosis de carga de $50 \mathrm{mg}$ de tigeciclina y luego $25 \mathrm{mg}$ cada $12 \mathrm{hr}$, por 7 a 14 días. Se consideraron elegibles aquellos pacientes cuyas infecciones eran profundas o requerían cirugía (ej. abscesos mayores, úlceras infectadas, quemaduras, mordeduras). Se excluyeron fascitis necrosantes, gangrenas, osteomielitis y otras infecciones que requerían tratamientos con un antibacteriano distinto al de estudio. También se excluyeron pacientes con insuficiencia renal, mujeres embarazadas o potenciales embarazadas, o con historia de hipersensibilidad a derivados de tetraciclina.

La población en el análisis de intención de tratamiento (ITT) incluyo 160 sujetos, 81 que recibieron tigeciclina $50 \mathrm{mg}$ y 79 que recibieron tigeciclina $25 \mathrm{mg}^{2}$. En lo basal, no existieron diferencias en edad, sexo, diagnostico primario o causa de la infección entre los dos grupos. Las infecciones más prevalentes fueron úlceras de pies infectadas, abscesos y mordeduras humanas o de animales. La población clínicamente evaluable incluyó 109 individuos. Se incluyeron 99 sujetos con cultivo basal positivo en el análisis microbiológico. En la visita de Test de Cura (TC), no hubo diferencias significativas de la tasa de curación clínica entre los grupos de la población clínicamente evaluable (CE) (74\% tigeciclina $50 \mathrm{mg}, 67 \%$ tigeciclina $25 \mathrm{mg}$ ). En forma similar, no hubo diferencias significativas en las tasas de erradicación microbiológica (69 y 56\%, respectivamente). Tigeciclina fue bien tolerada en ambos grupos, con tasas de reacciones adversas similares. Independiente de la dosis, los eventos adversos más comunes (menos de 5\%) fueron de índole gastrointestinal; las náuseas se reportaron en $28,1 \%$ de los sujetos $(34,6 \%$ tigeciclina $50 \mathrm{mg}, 21,5 \%$ tigeciclina $25 \mathrm{mg}$; p: NS), y vómitos en 5,6\% (18,5 y $12,7 \%$, respectivamente; p: NS). Dos pacientes de la rama de $50 \mathrm{mg}$ abandonaron el estudio por las náuseas y vómitos. Si bien ocurrieron cuatro muertes durante el estudio, ninguna de ellas se consideró relacionada con tratamiento (cuatro paros cardíacos y un cáncer de colon metastásico). Los autores concluyeron que tigeciclina fue eficaz y bien tolerada en el tratamiento de ICPTB.

Se han publicado en forma individual y conjunta ${ }^{10}$ dos estudios internacionales fase 3 , ambos de tipo aleatorios, prospectivos y doble ciego; los estudios ICPTB Tigeciclina $300^{3}$ e ICPTB Tigeciclina $305^{4}$. Estos estudios comparaban la eficacia y seguridad en ICPTB de una dosis de carga de $100 \mathrm{mg}$ iv de tigeciclina seguida de $50 \mathrm{mg}$ iv cada $12 \mathrm{hrs}$ versus vancomicina 1 g iv cada 12 hrs durante 5 a 14 días. El estudio ICPTB Tigeciclina 300 enroló 583 sujetos provenientes de 89 centros médicos en Norteamérica, Sudamérica e India y el estudio ICPTB tigeciclina 305 enroló 545 sujetos de 65 sitios en Europa, Asia, Australia y Sudáfrica.
Los sujetos fueron enrolados entre agosto de 2001 y febrero de $2004^{3,4,10}$ siendo elegibles los adultos sobre 18 años de edad con una ICPTB que involucrase tejidos profundos o un área extensa (mayor a $10 \mathrm{~cm}$ ), que requiriesen cirugía, o que se asociase a una patología subyacente significativa. Se excluyeron del análisis final los sujetos que no completaron al menos cinco días de terapia endovenosa o que recibieron más de dos dosis de agentes antimicrobianos no considerados en el estudio, incluyendo irrigaciones con antibacterianos tópicos.

Para mantener el tratamiento ciego, las bolsas y tubos de infusión se cubrieron con un color uniforme. Los sujetos asignados a tigeciclina recibían una infusión de solución salina isotónica además del fármaco de estudio para asimilarse al régimen de dos fármacos recibido por el brazo de vancomicina más aztreonam. Un profesional no ciego, independiente, ajustaba la dosis de vancomicina según función renal, no existiendo un protocolo de monitoreo de niveles de vancomicina pre-definido. Basados en el juicio clínico del investigador, la terapia con aztreonam podía ser discontinuada luego de dos días. Un investigador ciego monitorizó la evolución clínica de los pacientes con ICPTB y determinó el resultado clínico en la vista TC. El estudio fue diseñado para establecer una no inferioridad entre tigeciclina y sus comparadores (vancomicina más aztreonam). Si la diferencia de eficacia entre las ramas de tratamiento excedía el $15 \%$ usando el menor limite del intervalo de confianza, se podía considerar que los tratamientos satisfacían el criterio de no inferioridad. Se incluyó en el análisis de ITT al conjunto de 1.153 pacientes, de los cuales 1.116 calificaron para el análisis ITT modificado (ITTm), 1.057 para el análisis ITT clínico modificado (ITTc-m), 833 para el análisis de los CE y 540 para el de los microbiológicamente evaluables ${ }^{10}$. La razón más común para exclusión del análisis CE fue la violación del ciego (n: 80), falta de apego a criterios mínimos de ingreso (n: 60), pérdida de seguimiento/deserción a la visita TC (n: 55) y aislamiento de Pseudomonas aeruginosa en el cultivo basal (n: 7). En la población CE, el grupo conjunto de tigeciclina incluyó 422 sujetos y el grupo conjunto del comparador 411. Las características demográficas y clínicas de los dos grupos fueron similares. En globo, $20 \%$ de la población estudiada tenía diabetes mellitus y $7 \%$ enfermedad vascular periférica. Se consideró la infección espontánea en $50 \%$ de los casos, mientras que el trauma dio pie a la infección en 25 a $30 \%$ de los mismos. El diagnóstico primario fue, en casi $60 \%$ de la población estudiada, una infección de tejidos blandos profunda y en $28 \%$ un absceso mayor.

En el análisis conjunto de los tres estudios ${ }^{10}$, no hubo diferencias estadísticamente significativas en las 
tasas de curación clínica entre tigeciclina y vancomicina más aztreonam, ya sea en el análisis de $\operatorname{ITTm}(79,7$ y $81,9 \%$, respectivamente; $95 \% \mathrm{CI},-7,1$ a 2,8$)$ o de la población CE (86,5 y 88,6\%; 95\% CI, $-6,8$ a 2,7$)$.

El éxito del tratamiento no se vio afectado por el diagnostico clínico ni enfermedades subyacentes o patógeno causal (ej. $S$. aureus meticilina-sensible, $S$. aureus meticilina-resistente). En los evaluables microbiológicamente, las tasas de éxito fueron comparables para tigeciclina y vancomicina más aztreonam $(82,1$ y $86,2 \%$, respectivamente; $95 \% \mathrm{CI},-10,6$ a 2,4). Es interesante consignar que se identificaron 26 aislados de Bacteroides fragilis (13 en cada grupo) siendo incluidos en el análisis de las tasas de curación clínica, aun cuando vancomicina y aztreonam carecen de actividad intrínseca sobre anaerobios. Basados en los resultados de estos estudios, se concluyó que tigeciclina tenía una eficacia no inferior a vancomicina más aztreonam en el tratamiento de ICPTB ${ }^{10}$.

La incidencia global de eventos adversos asociados a tratamiento fue significativamente mayor en el grupo de tigeciclina comparado con vancomicina más aztreonam $(67,7 \text { vs } 61,1 \% \text {, respectivamente; } p=0,024)^{10}$. La mayoría de los eventos adversos reportados fueron de intensidad leve a moderada y se consideraron no relacionados con el tratamiento del estudio. En la población del análisis ITTm, experimentaron más eventos adversos gastrointestinales el grupo de tigeciclina versus vancomicina más aztreonam (45,6 vs 20,5\%, respectivamente; $\mathrm{p}<0,001)$ incluyendo náuseas $(34,5$ vs $3,6 \% ; \mathrm{p}<0,001)$, vómitos (19,6 vs 3,6\%; $<<0,001)$, diarrea $(8,5$ vs 5,1\%; p: NS), dispepsia $(3,7$ vs $0,9 \%$; p: $0,002)$, y anorexia $(3,4$ vs $0,4 \% ; \mathrm{p}<0,001)$. Si bien la mayoría de estos eventos fueron leves a moderados, siete pacientes en el grupo de tigeciclina abandonaron el estudio, seis con náuseas y uno con una úlcera duodenal perforada. Los eventos adversos relacionados a tratamiento reportados por los pacientes (menos de 5\%) incluyeron náuseas, vómitos en las tasas reportadas arriba, como también cefalea $(8,7 \%)$. De los siete pacientes fallecidos durante los estudios, seis $(85,7 \%)$ habían sido asignados, en forma aleatoria, para recibir tigeciclina. Los investigadores revisaron todos los casos y concluyeron que tales muertes no se relacionaban con la medicación de estudio ${ }^{3,4,10}$.

\section{Infecciones intra-abdominales complicadas (IIAC)}

La eficacia de tigeciclina en IIAC se estudió en dos ensayos multicéntricos fase 3 , aleatorios y doble ciego; el estudio Tigeciclina $301^{5}$ y Tigeciclina $306^{6}$. El primer estudio enroló 8.834 sujetos de 96 centros en Norteamérica, Latinoamérica, Europa y Asia. El segundo, tigeciclina 306, enroló 824 individuos de 94 sitios en Europa, Asia, Australia y Sudáfrica. Babinchak rea- lizó un análisis conjunto de eficacia y tolerabilidad para tigeciclina y sus comparadores en ambos estu$\operatorname{dios}^{11}$.

El enrolamiento se llevo a cabo entre noviembre de 2002 y mayo de $2004^{5,6,11}$. Los candidatos para el estudio fueron adultos a partir de los 18 años de edad, con una IIAC sospechada o confirmada (p. ej.: absceso secundario a apendicitis perforada, colecistitis, diverticulitis, peritonitis asociada a purulencia o contaminación fecal), que requiere cirugía. Criterios de exclusión incluían síndromes pancreáticos (pancreatitis aguda o abscesos pancreáticos), leucemia activa o recientemente tratada, cáncer metastásico, neutropenia, SIDA, enfermedad del SNC no controlada e infecciones abdominales no complicadas (peritonitis espontáneas, colecistititis, apendicitis sin evidencia de ruptura). Se excluyeron también los pacientes con un índice APACHE II mayor a 30; con enfermedad hepática grave, insuficiencia renal, aislado en cultivo basal de patógeno resistente a algunas de los fármacos de estudio ( $\mathrm{p}$. ej.: P. aeruginosa, Proteus mirabilis) o uso intraoperatorio de irrigaciones o esponjas con antimicrobianos.

Una vez estratificados por gravedad de su enfermedad (índice APACHE II menor de 15 o de 16 a 30), los sujetos fueron randomizados en una proporción 1:1 para recibir una dosis de carga de tigeciclina de $100 \mathrm{mg}$ iv seguida de $50 \mathrm{mg}$ cada $12 \mathrm{hrs}$ o imipenem/cilastatina $500 \mathrm{mg}$ cada 6 hrs (ajustado a función renal) por 5 a 14 días $^{5,6,11}$. Para mantener el estudio ciego, los individuos en la rama de tigeciclina recibían además dos infusiones de solución salina para simular las dosis de imipenem/cilastatina cada 6 hrs. Los fármacos del estudio se administraron cuando había alta sospecha clínica de IIAC o evidencia confirmatoria de una infección y junto a ello se obtenían cultivos basales.

Un investigador ciego monitorizaba periódicamente a los sujetos buscando presencia de fiebre, náuseas, vómitos y signos abdominales ${ }^{5,6,11}$. Se programó una visita TC dos a 42 días luego de completada la terapia del estudio para determinar el resultado del tratamiento.

Las mismas definiciones usadas en los estudios de las refs 3 y 4, se usaron para el análisis de ITT, análisis ITTm y población CE en los estudios de IIAC $^{5,6,11}$. Aquellos individuos de la población de análisis ITTm que satisfacían todos los criterios de inclusión, además de tener un cultivo basal positivo, constituían la población de análisis ITT microbiológica modificada (ITTm-m). Esta misma población se incluyó en el análisis ME si habían completado al menos cinco días de terapia, si no habían recibido más de dos dosis de fármacos no incluidos en el estudio, si tenían al menos un aislado que fuese susceptible a los medicamentos estudiadas y cumplieran con la visita TC. El objetivo 
primario a evaluar en estos estudio fue el éxito clínico a la visita TC en el análisis ITTm-m y en la población ME. El estudio fue diseñado para evaluar si tigeciclina no es inferior a imipenem/cilastatina para el tratamiento de IIAC. Si la diferencia de eficacia entre las ramas de tratamiento era igual o mayor a $15 \%$ usando el límite inferior del intervalo de confianza, el tratamiento se consideraba no inferior.

De los 1.658 pacientes randomizados, 1.642 (817 tigeciclina, 825 imipenem/cilastatina) recibieron tratamiento del estudio siendo incluidos en al análisis $\mathrm{ITTm}^{5,6,11}$. El análisis de CE, ITTm-m y ME incluyeron $1.382,1.262$ y 1.025 sujetos respectivamente. En el basal, los dos grupos no diferían significativamente en edad, sexo, raza, función renal o peso. En la población del análisis ITTm-m se incluyeron 631 pacientes en el grupo de tigeciclina y 631 en el de imipenem/cilastatina. Las causas más comunes de IIAC en ambos grupos fueron apendicitis $(50 \%)$ y colecistitis (14\%). El índice APACHE II promedio fue similar en los dos grupos (tigeciclina 6,3 e imipenem 6,0), si bien el grupo de tigeciclina contenía más pacientes con índice mayor a 15 (22 vs 13).

En la población $\mathrm{CE}$, la tasa de curación en la visita TC fue $86,7 \%$ para tigeciclina y $87,1 \%$ para imipenem/ cilastatina ( $95 \% \mathrm{CI},-4,1$ a 3,3; p: NS); las tasas correspondientes en la población ITTc-m fueron 79,8 y $82,0 \%$ $(95 \% \text { CI, -6,2 a 1,8; p: NS })^{11}$. No hubo diferencia significativa en las tasas de curación clínica entre las ramas de tratamiento en la población de análisis ITTm-m $(80,2$ y $81,5 \%$, respectivamente; $95 \%$ CI, $-5,8$ a 3,2$)$ y en la población ME (86,1 y 86,2\%; 95\% CI, -4,5 a 4,4). Algunos sujetos (40 tigeciclina/50 imipenem/cilastatina) desarrollaron bacteriemia como complicación de sus IIAC. En general, estos sujetos tuvieron resultado exitoso independiente del tratamiento recibido $(82,5 \mathrm{y}$ $80,0 \%$; 95\% CI, -16,0 a 19,6; p: NS). En la población ME no hubo diferencia significativa entre las tasas de erradicación de los brazos de tigeciclina y de imipenem/ cilastatina en general $(86,1$ y $86,2 \%$; $95 \%$ CI, $-4,5$ a 4,4$)$ en infección monomicrobiana ( 92,8 y $90,2 \%$; $95 \%$ CI, $3,7$ a 8,7$)$ o polimicrobiana $(82,5$ vs $83,7 \%$; $95 \%$ CI, $-7,1$ a 4,8). Tanto tigeciclina como imipenem/cilastatina tenían buenas tasas de erradicación contra Escherichia coli $(86,2$ y $87,1 \%)$ y Klebsiella pneumoniae $(88,5$ y $90,0 \%)$, los dos patógenos aislados más comúnmente (665 y 111 aislados respectivamente). Nótese que tigeciclina erradica el $80 \%(12 / 15)$ de los aislados productores de $\beta$-lactamasas de espectro extendido (BLEE) recuperados $(77,8 \%$ en $E$. coli [7/9] y $83,8 \%$ en $K$. pneumoniae [5/6]). Los resultados de estos ensayos sugieren que tigeciclina tiene una eficacia similar a imipenem/cilastatina en IIAC.

Se analizó para tolerabilidad a los pacientes de la población ITTm que recibieron 7 a 8 días de tratamiento ${ }^{11}$. Los tres eventos adversos más comúnmente reportados fueron náuseas $(24,4 \%$ con tigeciclina, 19\% con imipenem/cilastatina; $\mathrm{p}$ : 0,010), vómitos (19,2 versus $14,3 \%$ respectivamente; p: 0,008$)$ y diarrea $(13,8$ versus 13,2\%; p: NS). Ningún paciente en la rama de tigeciclina desarrolló diarrea asociada a Clostridium difficile o colitis durante los estudios. No se observó durante el estudio Tigeciclina-301 pacientes con prolongación significativa del intervalo QTc ${ }^{5}$. Hubo significativamente más abandonos de los estudios por eventos adversos en el grupo de tigeciclina versus el de imipenem/cilastatina (21/817 [2,6\%] versus $12 / 825$ [1,5\%], respectivamente; $\mathrm{p}$ : NS) ${ }^{11}$. Sólo se consideraron dos muertes relacionadas a tigeciclina. Ambos pacientes fueron enrolados en el estudio Tigeciclina301. Las muertes en ambos casos se atribuyeron a una respuesta inadecuada a tratamiento. Un paciente desarrollo un shock séptico luego de recibir tigeciclina por una semana y falleció dos días después de abandonar el estudio. El otro presentó sepsis después de tres días de terapia y falleció eventualmente de falla multiorgánica.

\section{Neumonía adquirida en la comunidad}

Durante el período junio de 2003 a julio de 2005 se efectuaron dos ensayos clínicos de tigeciclina en neumonía adquirida en la comunidad (NAC), recientemente comunicados $^{7}$ con vista a su licencia como indicación por parte de la Administración Federal de Drogas y Alimentos de E.U.A.- FDA. Se trata de los estudios Tigeciclina-308 y Tigeciclina-313, ambos ensayos fase 3 , multicéntricos, aleatorios y doble ciego. La información más relevante de estos estudios se resume en la Tabla 3.

En estos estudios se enrollaron 846 pacientes, que estuvieron disponibles para el análisis de ITTm, habiendo recibido todos al menos una dosis del fármaco en estudio. Al descartar los pacientes que no reunían criterios clínicos mínimos de NAC, se estableció la población del análisis ITTc-m. En la rama de tigeciclina para las poblaciones así descritas se incluyeron 424 y 394 pacientes, respectivamente. En la rama comparadora con levofloxacina se incluyeron 422 y 403 pacientes en forma respectiva. Finalmente, la población $\mathrm{CE}$ fue aquella que completó visita de TC con éxito o fracaso, excluidos los indeterminados, y que permaneció frente al equipo tratante como ciega, a lo largo de todo el estudio. En esta última segmentación se incluyeron 282 pacientes para tigeciclina y 292 para levofloxacina.

La dosis diaria de tigeciclina fue la estándar de carga y mantención. La de levofloxacina fue de $500 \mathrm{mg}$ iv diarios, en un estudio y de $500 \mathrm{mg}$ iv cada 12 ó $24 \mathrm{hrs}$ en otro, según practicas discrecionales de cada cen- 


\begin{tabular}{|c|c|c|c|c|c|}
\hline Estudio & Diseño & $\begin{array}{c}\text { n de } \\
\text { pacientes }\end{array}$ & Regímenes & Tasas de curación & $\begin{array}{l}\text { Efectos adversos más frecuentemente } \\
\text { reportados (> 5\%). Observaciones }\end{array}$ \\
\hline \multirow[t]{2}{*}{$\begin{array}{l}\text { Tanaseanu } \\
\text { y cols (7) }\end{array}$} & \multirow[t]{2}{*}{ Fase $3, M C, A L, D C$} & 424 & $\begin{array}{l}\text { TGC dosis de carga } \\
100 \mathrm{mg} \text { iv y luego } \\
50 \mathrm{mg} \mathrm{c} / 12 \mathrm{hrs}\end{array}$ & $\begin{array}{l}\text { CE } 89,7 \% \\
\text { ITTC-m } 81,0 \%\end{array}$ & $\begin{array}{l}\text { Cualquiera }(47,9 \%) \text {, náuseas }(20,8 \%) \text {, vómitos } \\
(13,2 \%) \text {, diarrea }(7,5 \%)\end{array}$ \\
\hline & & 422 & $\begin{array}{l}\text { Levofloxacina } 500 \text { mg } \\
\text { iv día (TGC 308) o } \\
\text { Levofloxacina } 500 \text { mg } \\
\text { iv c/12 hrs ó c/24 hrs, } \\
\text { según el criterio del } \\
\text { investigador (TGC 313) }\end{array}$ & $\begin{array}{l}\text { CE } 86,3 \% \\
\text { ITTC-m } 79,7 \%\end{array}$ & $\begin{array}{l}\text { Cualquiera }(37,4 \%) \text {, náuseas }(6,6 \%) \text {, diarrea }(5,5 \%) \text {, } \\
\text { elevación transaminasas AST }(6,4 \%) \text { ALT }(5,9 \%)\end{array}$ \\
\hline \multirow[t]{2}{*}{ Dartois y cols (8) } & \multirow[t]{2}{*}{ Fase $3, M C, A L, D C$} & 22 & $\begin{array}{l}\text { TGC dosis de carga } \\
100 \mathrm{mg} \text { iv y luego } \\
50 \mathrm{mg} \mathrm{c} / 12 \mathrm{hrs}\end{array}$ & $\begin{array}{l}90,9 \%(95 \% \quad I C \\
70,8-98,9)\end{array}$ & $\begin{array}{l}\text { Población microbiológicamente evaluable con } \\
\text { bacteriemia por S. pneumoniae }\end{array}$ \\
\hline & & 18 & $\begin{array}{l}\text { Levofloxacina } 500 \text { mg } \\
\text { iv día o levofloxacina } \\
500 \text { mg iv c/12 hrs ó } \\
\text { c/24 hrs, según el } \\
\text { criterio del investigador }\end{array}$ & $\begin{array}{l}72,2 \%(95 \% \quad I C \\
46,5-90,3)\end{array}$ & \\
\hline
\end{tabular}

tro. Según ese mismo criterio, ciertos pacientes con al menos tres días de terapia iv cumplida y evidenciando mejoría clínica, podían pasar a tratamiento oral con levofloxacina. En ambos estudios la duración del tratamiento era de 7 a 23 días. El TC se efectuó entre los 7 y 23 días posteriores a la finalización de los antibacterianos.

En la población ITTm (846 pacientes) las características demográficas, de evaluación clínica y co-morbilidades basales eran similares en ambas ramas de estudio. Casi la mitad de los pacientes tuvo un índice de gravedad PSI de Fine entre III y V (46,5\% tigeciclina; $47,2 \%$ levofloxacina). La duración media de la terapia fue 10 días para cada rama del estudio. En el estudio Tigeciclina-308, 124/138 (89,9\%) pacientes bajo tigeciclina cambiaron a terapia oral y lo que ocurrió en 137/156 (87,9\%) bajo levofloxacina; el número de días promedio para tal cambio fue 3,9 y 3,3 días, respectivamente. Estas diferencias no fueron estadísticamente significativas.

Para la población CE tampoco hubo diferencias en la tasa de curación entre tigeciclina $(253 / 282 ; 89,7 \%)$ y levofloxacina $(252 / 292 ; 86,3 \%)$. En la población ITTc-m la tasa de curación fue de 321/394 (81,0\%) en la rama de tigeciclina, no estadísticamente diferente a la del grupo de levofloxacina con 321/403 (79,7\%). El análisis estadístico demostró que tigeciclina no fue inferior a levofloxacina $(\mathrm{p}<0,001)$.

Un análisis del subgrupo de neumonías neumo- cóccicas bacteriémicas ${ }^{8}$, mostró tasas de curación de 20/22 (90,9\%; 95\% CI 70,8-98,9) para tigeciclina y de 13/18 (72,2\%; 95\% CI, 46,5-90,3) para levofloxacina.

No hubo diferencias entre ambos grupos en términos de duración de la estadía hospitalaria (media de días para tigeciclina 9,8 vs levofloxacina 9,8 días) ni en la media de días de tratamiento $(9,8$ vs 10 días, respectivamente).

Respecto de eventos adversos asociados a la medicación de estudio, hubo una significativa mayor ocurrencia en el grupo de tigeciclina versus levofloxacina $(47,9$ vs $37,4 \%, \mathrm{p}<0,01)$. Siendo la presencia de náuseas $(20,8$ vs 6,6\%) y vómitos (13,2 vs 3,3\%) significativamente mayores para tigeciclina que levofloxacina $(p<0,001)$. A pesar de esto, la tasa de descontinuación debida a eventos adversos fue similar para tigeciclina (n: 26, 6,1\%) y levofloxacina (n: $34,8,1 \%$ ). Los eventos adversos graves tuvieron similar frecuencia en los grupos estudiados $(9,9$ vs 10,9\%). El número de muertes también fue similar en ambos grupos (12 vs 11 pacientes, respectivamente), no atribuyéndose ninguna de estas a la medicación de estudio.

Los autores concluyen que tigeciclina es eficaz, con tasas de curación similar y no inferior a levofloxacina en pacientes hospitalizados por NAC, alcanzando buenas tasas de curación en pacientes con co-morbilidades, factores de gravedad y bacteriemia ${ }^{7}$. A pesar de la mayor incidencia de efectos adversos asociados 
al fármaco ello no redunda en la tasa de descontinuación, eventos adversos graves o muerte.

\section{Estudios clínicos no controlados}

Una vez disponible tigeciclina para el uso general, se han comunicado diversas experiencias clínicas que consideran tanto las indicaciones licenciadas como las no licenciadas, tratándose fundamentalmente de series de casos clínicos en el contexto de infecciones graves manejadas en cuidados críticos y/o producidas por agentes bacterianos multi-resistentes. Al respecto, detallaremos someramente la información comunicada al momento de establecer la presente revisión sistemática de la literatura. Dicha información y sus diferentes indicaciones se resumen en la Tabla 4 .

Muñoz-Price ${ }^{12}$ comunicó cuatro casos de pacientes con infecciones invasoras por Staphylococcus aureus meticilina resistentes (SAMR) que recibieron tratamiento con tigeciclina, luego del intento fracasado de terapia con antimicrobianos convencionales. Tres de ellos experimentaron bacteriemia por SAMR bajo hemodiálisis y un cuarto un empiema pleural bilateral. Las combinaciones de agentes antimicrobianos usados previamente a tigeciclina en los distintos pacientes fueron vancomicina + quinupristina/dalfopristina, vancomicina + daptomicina + linezolid, vancomicina + daptomicina y vancomicina + linezolid. Todas las cepas de SAMR estudiadas se mostraron susceptibles, tanto a los agentes ya mencionados como a tigeciclina. La respuesta clínica y bacteriológica a tigeciclina fue favorable en los cuatros pacientes; sin embargo, uno de ellos experimentó recaída de la bacteriemia por SAMR debido a una transitoria reducción de la dosis diaria del fármaco a la mitad.

Denton $^{13}$, reportó su experiencia inicial en el uso de tigeciclina en pacientes con patógenos respiratorios "difíciles" en fibrosis quística. Los patógenos tratados fueron Burkholderia cepacia, Pseudomonas apista, Stenotrophomonas maltophilia, Mycobacterium abscessus y Mycobacterium chelonae. Las alternativas terapéuticas de los seis pacientes eran limitadas dada la multi-resistencia o alergia a terapias convencionales. El autor consiguió respuesta clínica en cuatro de los seis pacientes donde se usó el antimicrobiano en combinación con $\beta$ lactámicos y aminoglucósidos. Dos pacientes discontinuaron el tratamiento por náuseas y vómitos.

Chemaly ${ }^{14}$, reportó una serie de 38 pacientes oncológicos con neumonía refractaria que recibieron tigeciclina durante al menos $72 \mathrm{hrs,} \mathrm{luego} \mathrm{de} \mathrm{fracasar} \mathrm{la}$ terapia antimicrobiana de primera línea en 36 casos y en dos debido a alergia a vancomicina $u$ otro $\beta$ - lactámico. Catorce pacientes habían sido sometidos a trasplante de precursores hematopoyéticos (TPH). Junto a tigeciclina se ofreció un antimicrobiano con acción anti-pseudomónica. Sólo 10 (26\%) pacientes contaban con agente bacteriano aislado en cultivos, principalmente bacterias multi-resistentes. Del total de pacientes, 63\% (24/38) evidenció respuesta clínica favorable, el restante $37 \%$ falleció. En el grupo con etiología demostrada 8 de $10(80 \%)$ respondieron a terapia. Un paciente presentó náuseas y otro diarrea. Se concluyó que el uso de tigeciclina más un agente con cobertura anti-pseudomonas parece una adecuada elección de terapia en pacientes oncológicos, con o sin TPH.

Schafer ${ }^{15}$, notificó el uso inicial de tigeciclina sola o combinada con otros antimicrobianos en pacientes con neumonía asociada a ventilación mecánica (NAVM) y/o bacteriemia debida a Acinetobacter baumannii multiresistente. De los 25 pacientes incluidos, 19 tenían NAVM, tres NAVM más bacteriemia y dos sólo bacteriemia. En 23/25 de las cepas aisladas se evidenció susceptibilidad reducida a imipenem/cilastatina y sólo en uno resistencia a tigeciclina. Sólo cinco de ellos recibieron monoterapia con tigeciclina, en el resto de los pacientes se combinó con los siguientes antibacterianos: imipenem/cilastatina iv nueve, colistín nebulizado seis, imipenem/cilastatina iv más colistín nebulizado tres, colistín iv uno e imipenem/cilastatina más colistín iv uno. En 21/25 (84\%) pacientes se consiguió resolución clínica de su infección, demostrándose erradicación microbiológica en 12/15 (80\%) pacientes donde se obtuvo cultivos de seguimiento.

Otra comunicación de Lewis ${ }^{16}$, reportó nueve pacientes de cuidados críticos, que recibieron más de 24 horas de tratamiento con tigeciclina en un contexto clínico de uso restringido del agente antimicrobiano para agentes gramnegativos multi-resistentes, SAMR y/o Enterococcus resistentes a vancomicina (ERV). Sólo tres de estos pacientes experimentaron una respuesta favorable a la terapia, los otros seis mostraron infección persistente. En este último grupo cuatro casos correspondían a NAVM y dos a bacteriemia. Se cambió el tratamiento antibacteriano a cuatro de seis, falleciendo y abandonando tratamiento los restantes. Se observó aumento de la CIM a tigeciclina en dos cepas estudiadas, una SAMR y otra ERV.

En un reporte de casos prospectivo, Burgos y cols ${ }^{17}$, incluyeron 27 casos de infecciones por $K$. pneumoniae multi-resistente portadora de carbapenemasa, solamente sensible a polimixina B. De estas infecciones 11 eran urinarias, nueve respiratorias, seis bacteriemias y una biliar. Los autores consiguieron respuesta clínica positiva en $15 / 27$ casos $(55 \%)$ negativa en $11 / 27(41 \%)$ e indeterminada en uno. Se 
Tabla 4. Resumen de reportes de uso de tigeciclina en series clínicas, según indicación

\begin{tabular}{|c|c|c|c|c|c|}
\hline Estudio & $\begin{array}{l}\text { Tipo de estudio } \\
\text { e Indicación }\end{array}$ & $\begin{array}{c}n \text { de } \\
\text { pacientes }\end{array}$ & Regímenes & Tasas de curación & $\begin{array}{l}\text { Efectos adversos más frecuentemente } \\
\text { reportados }(>5 \%) \text {. Comentarios }\end{array}$ \\
\hline $\begin{array}{l}\text { Muñoz-Price } \\
\text { y cols (12) }\end{array}$ & $\begin{array}{l}\text { Serie de casos, } \\
\text { infecciones invasoras } \\
\text { por SAMR }\end{array}$ & $\begin{array}{l}4 \\
\text { (3 hemodiálisis, } \\
1 \text { empiema) }\end{array}$ & $\begin{array}{l}\text { TGC dosis de carga } \\
100 \mathrm{mg} \text { iv y luego } \\
50 \mathrm{mg} \mathrm{c} / 12 \mathrm{hrs}\end{array}$ & $100 \% \quad(4 / 4)$ & $\begin{array}{l}\text { Fracaso previo de vancomicina, linezolid, } \\
\text { quinupristina/dalfopristina } y / o \text { daptomicina }\end{array}$ \\
\hline $\begin{array}{l}\text { Denton } \\
\text { y cols (13) }\end{array}$ & $\begin{array}{l}\text { Series de casos, } \\
\text { Fibrosis quística }\end{array}$ & 6 & $\begin{array}{l}\text { TGC dosis de carga } \\
100 \mathrm{mg} \text { iv y luego } \\
50 \mathrm{mg} \mathrm{c} / 12 \text { hrs }+ \\
\beta \text {-lactámico y/o } \\
\text { aminoglucósido }\end{array}$ & $66 \%(4 / 6)$ & $\begin{array}{l}\text { Náuseas y vómitos (33\%), } 2 \text { pacientes } \\
\text { discontinúan }\end{array}$ \\
\hline $\begin{array}{l}\text { Chemaly } \\
\text { y cols (14) }\end{array}$ & $\begin{array}{l}\text { Serie de casos, } \\
\text { neumonía nosocomial } \\
\text { en pacientes con } \\
\text { neoplasias hemato- } \\
\text { lógicas, c/s TPH }\end{array}$ & $\begin{array}{c}38 \\
\text { (36 en fracaso } \\
\text { 1era línea, } \\
2 \text { por alergia a } \\
\text { otros fármacos) }\end{array}$ & $\begin{array}{l}\text { TGC dosis de carga } \\
100 \mathrm{mg} \text { iv y luego } \\
50 \mathrm{mg} \mathrm{c/12} \mathrm{hrs}+ \\
\text { Anti-pseudomónico }\end{array}$ & $63 \%(24 / 38)$ & $\begin{array}{l}\text { Náuseas }(1 / 38) \text {, diarrea }(1 / 38) \\
14 / 38 \text { pacientes fallecen }\end{array}$ \\
\hline \multirow[t]{2}{*}{$\begin{array}{l}\text { Schafer } \\
\text { y cols (15) }\end{array}$} & $\begin{array}{l}\text { Series de casos, } \\
\text { NAVM y/o bacteriemia } \\
\text { por A. baumannii } \\
\text { multi-resistente }\end{array}$ & $\begin{array}{l}25 \\
\text { (19 NAVM, } \\
3 \text { bacteriemia, } \\
3 \text { ambas) }\end{array}$ & $\begin{array}{l}\text { TGC dosis de carga } \\
100 \mathrm{mg} \text { iv y luego } \\
25-50 \mathrm{mg} \text { diarios }\end{array}$ & $\begin{array}{l}\text { Resolución clínica } \\
84 \%(21 / 25)\end{array}$ & $\begin{array}{l}\text { Sin EA evaluables pues los pacientes estaban } \\
\text { intubados }\end{array}$ \\
\hline & & & $\begin{array}{l}\text { C/S } \\
\text { Colistín NBZ y/o } \\
\text { imipenem/cilastatina } \\
\text { iv y/o colistín iv }\end{array}$ & $\begin{array}{l}\text { Resolución microbio- } \\
\text { lógica } 48 \%(12 / 25)\end{array}$ & $\begin{array}{l}\text { 23/25 aislados de } A \text {. baumannii con susceptibili- } \\
\text { dad reducida a imipenem/cilastatina, 1/25 no } \\
\text { susceptible a TGC }\end{array}$ \\
\hline $\begin{array}{l}\text { Lewis } \\
\text { y cols (16) }\end{array}$ & $\begin{array}{l}\text { Series de casos, } \\
\text { pacientes en cuidados } \\
\text { críticos }\end{array}$ & 9 & $\begin{array}{l}\text { TGC dosis de carga } \\
100 \mathrm{mg} \text { iv y luego } \\
50 \mathrm{mg} \mathrm{c} / 12 \mathrm{hrs}\end{array}$ & $\begin{array}{l}\text { Evolución favorable } \\
33 \%(3 / 9) \\
\text { No favorable } 66 \%(6 / 9)\end{array}$ & $\begin{array}{l}\text { Infecciones de evolución desfavorable: } \\
\text { neumonía } 4 / 6 \text {, bacteriemia } 2 / 6\end{array}$ \\
\hline $\begin{array}{l}\text { Burgos } \\
\text { y cols (17) }\end{array}$ & $\begin{array}{l}\text { Serie de casos, } \\
\text { prospectivo, infección } \\
\text { por } K \text {. pneumoniae } \\
\text { carbapenemasa }(+) \text {. }\end{array}$ & $\begin{array}{l}27 \\
\text { (6 bacteriemias, } \\
9 \text { respiratorias, } \\
11 \text { ITU, } 1 \text { biliar) }\end{array}$ & $\begin{array}{l}\text { TGC dosis de carga } \\
100 \mathrm{mg} \text { iv y luego } \\
50 \mathrm{mg} \mathrm{c} / 12 \mathrm{hrs}\end{array}$ & $\begin{array}{l}\text { Respuesta clínica } 55 \% \\
(15 / 27) \text {, microbioló- } \\
\text { gica } 75 \%(12 / 16)\end{array}$ & Náuseas $(33 \%)$, vómitos $(22 \%)$, diarrea $(11 \%)$ \\
\hline $\begin{array}{l}\text { Leclerc } \\
\text { y cols (18) }\end{array}$ & $\begin{array}{l}\text { Serie de casos, } \\
\text { infecciones pulmonares } \\
\text { por } A \text {. baumannii } \\
\text { en } \cup C l\end{array}$ & 6 & $\begin{array}{l}\text { TGC dosis de carga } \\
100 \mathrm{mg} \text { iv y luego } \\
50 \mathrm{mg} \mathrm{c} / 12 \mathrm{hrs}\end{array}$ & $\begin{array}{l}\text { Resolución clínica } \\
50 \%\end{array}$ & $\begin{array}{l}\text { Dos casos inicialmente susceptibles desarrollan } \\
\mathrm{CIM}>4 \mu \mathrm{g} / \mathrm{ml} \text { intra-tratamiento }\end{array}$ \\
\hline $\begin{array}{l}\text { Muñoz Price } \\
\text { y cols (19) }\end{array}$ & $\begin{array}{l}\text { Serie retrospectiva, } \\
\text { bacteriemias por } \\
\text { A. baumannii multi- } \\
\text { resistente }\end{array}$ & $\begin{array}{l}14 \\
\text { (8 reciben TGC, } \\
6 \text { reciben } \\
\text { colistín) }\end{array}$ & $\begin{array}{l}\text { TGC dosis de carga } \\
100 \mathrm{mg} \text { iv y luego } \\
50 \mathrm{mg} \text { c/12 hrs vs } \\
\text { colistín iv } 5 \mathrm{mg} / \mathrm{kg} / \text { día }\end{array}$ & $\begin{array}{l}\text { n de días para negati- } \\
\text { vizar hemocultivos; } \\
\text { TGC } 6,2 \text { vs colistín } 3\end{array}$ & $\begin{array}{l}\text { Hubo bacteriemia (2/8) intra-tratamiento en grupo } \\
\text { de TGC, todas las cepas chequeadas como sen- } \\
\text { sibles a TGC }\end{array}$ \\
\hline $\begin{array}{l}\text { Swoboda } \\
\text { y cols (20) }\end{array}$ & $\begin{array}{l}\text { Serie de casos, sepsis } \\
\text { grave o shock séptico }\end{array}$ & $\begin{array}{c}70 \\
\text { (35 infección } \\
\text { abdominal, } \\
48+\text { otro foco, } \\
12 \text { neumonía, } \\
22+\text { otro foco, } \\
10 \text { infección } \\
\text { abdominal + } \\
\text { neumonía) }\end{array}$ & 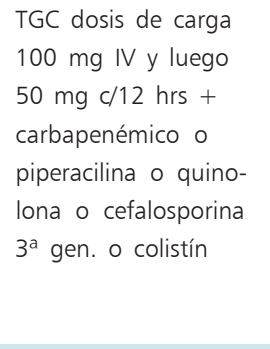 & $\begin{array}{l}\text { Mortalidad } \\
30 \%\end{array}$ & $\begin{array}{l}\text { Etiología predominante fueron grampositivos } \\
(72 \%) \text {, fundamentalmente especies de } \\
\text { Enterococcus sp }(66 \%)\end{array}$ \\
\hline $\begin{array}{l}\text { Paranythiotou } \\
\text { y cols }(21)\end{array}$ & $\begin{array}{l}\text { Serie de casos, } \\
\text { infecciones por } \\
\text { agentes multi-resistente } \\
\text { en UCI }\end{array}$ & $\begin{array}{l}18 \\
(11 \text { NAVM, } \\
4 \text { misceláneas, } \\
3 \text { empírico) }\end{array}$ & $\begin{array}{l}\text { TGC } 50-100 \mathrm{mg} \text { iv } \\
\mathrm{c} / 12 \mathrm{hrs} \\
\mathrm{C} / \mathrm{S} \\
\text { colistín } \mathrm{y} / \mathrm{o} \\
\text { meropenem } \mathrm{y} / \mathrm{u} \text { otro }\end{array}$ & $\begin{array}{l}\text { NAVM } \\
\text { Resolución clínica y } \\
\text { microbiológica } \\
90,1 \%(10 / 11) \\
\text { Mortalidad } 45,5 \%\end{array}$ & $\begin{array}{l}\text { En uso empírico mortalidad } 100 \% \text {, otras infec- } \\
\text { ciones no NAVM, } 75 \% \text {. }\end{array}$ \\
\hline
\end{tabular}


dispuso de datos para evaluar la respuesta microbiológica en 16 casos (59\%), de éstos 12 (75\%) tuvieron respuesta positiva y cuatro $(25 \%)$ negativa. La presencia de eventos adversos gastrointestinales fue consistente a lo reportado en la literatura médica, a saber náuseas $33 \%$, vómitos $22 \%$ y diarrea $11 \%$.

Dos reportes del ICAAC 2007 abordan el uso de tigeciclina en infecciones por $A$. baumanni resistente a carbapenémicos; Leclerc $^{18}$, informó un brote de infecciones respiratorias por tal agente; de los seis pacientes estudiados sólo la mitad consiguió resolución clínica favorable, dos de las cepas inicialmente susceptibles desarrollaron resistencia durante el tratamiento con tigeciclina (CIM $>4 \mu \mathrm{g} / \mathrm{ml}$ ). Muñoz-Price ${ }^{19}$, a su vez, reportó que en 14 bacteriemias, manejadas en ocho casos con tigeciclina y en seis con colistín, el número de días requeridos para conseguir negativización de hemocultivos fue mayor en el grupo de tigeciclina (6,2 días) versus colistín (3 días). Todas las cepas fueron susceptibles a tigeciclina. Sin embargo, dos pacientes que recibían el fármaco por otra indicación desarrollaron bacteriemia por $A$ baumannii. Esta información sugiere que frente a infecciones por tal agente, aun cuando tigeciclina parece ser útil, puede inducir la aparición de resistencia durante la terapia, por mecanismos que deben ser bien estudiados.

Más recientemente, Swoboda y cols ${ }^{20}$, evaluaron el uso del medicamento en una unidad de cuidados críticos quirúrgica, en 70 pacientes con sepsis grave o shock séptico. La mayoría de los pacientes tenía co-morbilidades como cáncer $(51 \%)$ o falla renal que requería terapia de reemplazo $(57 \%)$. El índice APACHE II al ingreso promedio fue $27 \pm 9,7$ (rango 7-52). La indicación clínica para el uso de tigeciclina fue infección abdominal complicada exclusiva en 35 pacientes $(50 \%) \mathrm{y} / \mathrm{o}$ combinada con otro foco en 48 pacientes $(69 \%)$, neumonía exclusiva en $12(17 \%)$ y/o combinada con otro foco en 22 (31\%), infección abdominal más neumonía en 10 pacientes (14\%) e infección de piel y tejidos blandos complicada en tres pacientes $(4 \%)$, entre los cuadros más relevantes. Hubo una clara predominancia de cocáceas grampositivas como agentes asociados a la infección (66\%), a saber, Enterococcus faecium 26 (37\%), ERV 15 (21\%), Enterococcus faecalis 7 (10\%), SAMR 4 (6\%) y otros Enterococcus $4(6 \%)$. También se aisló $S$. maltophilia en 13 pacientes (19\%), en 11 casos $(16 \%)$ no se documentó el origen de la infección. Tigeciclina se utilizó como régimen de segunda línea en las dosis estándares, siendo el tiempo promedio de $9 \pm 4$ días. Sólo en 15 casos (21\%) se utilizó como monoterapia; en 29 se asoció a un carbapenémicos, en cinco a piperacilina, en cinco a fluoroquinolonas, en tres a colistín y en tres a una cefalosporina de $3^{\mathrm{a}}$ genera- ción, entre otros. La mortalidad de la serie estudiada fue de $30 \%$, siendo predictores de mortalidad en el análisis multivariado el índice APACHE II y la terapia de reemplazo renal.

Paramythiotou ${ }^{21}$, presentó otra serie prospectiva de pacientes quirúrgicos en cuidados críticos con infecciones debidas a patógenos multi-resistentes. Se incluyeron 18 pacientes con infecciones graves de riesgo vital, que no respondieron a terapia previa o estaban colonizados con patógenos pan-resistentes. Once pacientes padecían de NAVM, cuatro de infecciones misceláneas y tres fueron sometidos a terapia empírica, sin sostén microbiológico. Los agentes bacterianos rescatados en 11 casos fueron $A$. baumannii (10 en NAVM), cinco $K$. pneumoniae productora de BLEE, una $P$. aeruginosa productora de carbapenemasa y un ERV. Dos cepas de $A$. baumannii se mostraron resistentes a tigeciclina (CIM $>4 \mathrm{mg} / \mathrm{l})$. La duración promedio de la terapia con tigeciclina fue de 15,8 días (rango 10-24) en NAVM, 25 días en misceláneas (rango 10-43) y siete días (rango 4-10) en el grupo de terapia empírica. El promedio de la serie total fue de 16,4 días. La dosis diaria de tigeciclina iv fue de 50 a $100 \mathrm{mg} / 12 \mathrm{hrs}$. Siete pacientes recibieron tigeciclina como monoterapia, cuatro asociada a colistín, cuatro junto a colistín y meropenem, uno junto a ciprofloxacina, otro junto a colistín más linezolid y un último junto a colistín y teicoplanina. Se consiguió respuesta clínica y microbiológica en 10/11 pacientes con NAVM, 4/4 y 3/4 respectivamente en las infecciones miscelánea y ninguna en el grupo de terapia empírica. Sólo 1/11 presentó mortalidad a los 14 días en NAVM, pero $5 / 11$ fallecieron tardíamente. En misceláneas fallecieron 1/4 a los 14 días y 3/4 tardíamente. En los de manejo empírico estaban fallecidos todos a los 14 días sin evidenciar respuesta clínica. Los autores destacan la utilidad de tigeciclina en el tratamiento de la NAVM, siendo tigeciclina una alternativa eficaz que supera el problema de resistencia bacteriana en cuidados críticos.

\section{Reporte de casos aislados}

Varios autores reportan casos anecdóticos con uso de tigeciclina en los más variados contextos: endocarditis por ERV resistente a linezolid, cloranfenicol y quinupristina/dalfopristina ${ }^{22}$, bacteriemia por $K$. pneumoniae BLEE $(+)$ y carbapenemasa $(+)^{23}$; peritonitis por SAMR secundaria a peritoneodiálisis ${ }^{24}$; sepsis recurrente de foco urinario por E. coli BLEE $(+)$ ante fracaso de meropenem ${ }^{25}$; shock séptico por A. baumannii pan resistente ${ }^{26}$; neumonía nosocomial por SAMR 
en paciente sometido a trasplante hepático con fracaso de vancomicina y linezolid ${ }^{27}$; absceso pulmonar y empiema por $K$. pneumoniae carbapenemasa $(+)^{28}$; osteomielitis por $P$. aeruginosa en paciente sometido a $\mathrm{TPH}^{29}$, osteomielitis por Mycobacterium fortuitum en en paciente sometido a $\mathrm{TPH}^{30}$ y shock séptico debido a infección por ERV ${ }^{31}$.

\section{Eventos adversos}

Los datos de tolerabilidad sometidos a consideración de la FDA como parte de su proceso de registro consistían en una información conjunta de los estudios fase 3 efectuados en ICPTB $^{3,4}$ y en IIAC ${ }^{5,6}$ más datos de un estudio comparativo con linezolid en infecciones graves causadas por ERV no publicado ${ }^{32}$, que representan una población conjunta de 1.415 pacientes. A estos pacientes habría que sumar lo observado en 424 pacientes incluidos en el análisis ITTm de los estudios fase 3 en $\mathrm{NAC}^{8}$. El resumen de los eventos adversos asociados a tratamiento en pacientes tratados con tigeciclina de todos estos estudios se muestra en la Tabla 5. En el análisis global claramente fueron predominante los eventos adversos gastrointestinales, tal como náuseas $(27,5 \%)$, vómitos $(18,2 \%)$ y diarrea $(11,5 \%)$, luego un poco menos frecuentes las reacciones en el sitio de infusión $(7,5 \%)$ y la cefalea $(3,5 \%)$

En los estudios en ICPTB e IIAC 1.383 sujetos recibieron al menos una dosis de tigeciclina. Sólo 58 $(4,2 \%)$ discontinuaron tigeciclina a raíz de un evento adverso, siendo más común la presencia de náuseas y vómitos $\left(\chi^{2}\right)$. De los 30 (2,2\%) fallecidos en estos estudios, sólo dos muertes presumiblemente se asociaron a la medicación de estudio. En los estudios de $\mathrm{NAC}^{8}$ $203(47,9 \%)$ pacientes reportaron eventos adversos; sin embargo, sólo 12 casos (2,8\%) discontinuaron terapia en número similar al grupo comparador. No hubo muertes asociadas a la medicación de estudio.

\section{Discusión}

La prevalencia de infecciones nosocomiales por agentes bacterianos multi-resistentes muestra una tendencia creciente en las últimas décadas, especialmente bacterias gramnegativas portadoras de BLEE y/o carbapenemasa. Por otro lado, el desarrollo de nuevas clases de antimicrobianos con cobertura específica sobre tales agentes está lejos de ser adecuada. La introducción de tigeciclina, la primera glicilciclina, podría proveernos de una opción adicional para el manejo de estas complejas infecciones. Así parece mostrarlo la evidencia revisada de su uso inicial en ensayos clínicos y series de casos de "la vida real".

\section{Tabla 5. Frecuencia reportada $(\geq 3,5 \%)$ de efectos adversos asociados a} terapia con tigeciclina en estudios fase III (Total $n=1.839$ )

\begin{tabular}{|c|c|c|c|}
\hline $\begin{array}{l}\text { Evento } \\
\text { adverso }\end{array}$ & $\begin{array}{l}\text { de ICPTB e IIAC } 3-6,32 \\
\text { N: } 1.415\end{array}$ & $\begin{array}{c}\text { Estudios de } \mathrm{NAC}^{7,8} \\
\text { N: } \mathbf{4 2 4}\end{array}$ & $\begin{array}{c}\text { Total } \\
\text { N: } 1.839\end{array}$ \\
\hline Generales & $\%$ & $\%$ & $\%$ \\
\hline - Infección & 8,3 & - & - \\
\hline - Fiebre & 7,1 & - & - \\
\hline - Dolor abdominal & 6,8 & - & - \\
\hline - Cefalea & 5,9 & 3,5 & 5,3 \\
\hline - Dolor & 3,7 & - & - \\
\hline \multicolumn{4}{|l|}{ Cardiovascular } \\
\hline - Hipertensión arterial & 4,9 & - & - \\
\hline \multicolumn{4}{|l|}{ Gastrointestinales } \\
\hline - Náuseas & 29,5 & 20,8 & 27,5 \\
\hline - Vómitos & 19,7 & 13,2 & 18,2 \\
\hline - Diarrea & 12,7 & 7,5 & 11,5 \\
\hline \multicolumn{4}{|l|}{ Metabólicos } \\
\hline - Halitosis & 3,5 & - & - \\
\hline \multicolumn{4}{|l|}{ SNC } \\
\hline - Mareos & 3,5 & - & - \\
\hline \multicolumn{4}{|l|}{ Respiratorios } \\
\hline - Aumento de la tos & 3,7 & - & - \\
\hline \multicolumn{4}{|l|}{ Otros } \\
\hline - Flebitis & 9,0 & 4,0 & 7,8 \\
\hline
\end{tabular}

ICPTB: infecciones complicadas de piel y tejidos blandos; IIAC: infecciones intra-abdominales complicadas; NAC: neumonía adquirida en la comunidad.

Además de la bien demostrada eficacia de tigeciclina en ICPTB e IIAC ${ }^{3,4}$, indicaciones ya licenciadas del fármaco, se espera que prontamente se autorice su indicación en NAC. Sin embargo, probablemente su nicho real de utilización estaría orientado hacia el manejo de infecciones del ámbito intrahospitalario, sobre todo en el contexto de cuidados críticos. Según la información revisada, se insinúa una gran utilidad en el manejo de $\mathrm{NAVM}^{15,21}$ y en sepsis graves $^{20}$; para asentar una posición sólida al respecto deberemos esperar los resultados de los estudios controlados actualmente en curso para tales indicaciones.

Por lo demás, si bien el fármaco muestra un perfil de eventos adversos gastrointestinales algo frecuentes, a pesar de ello, es bien tolerado y son escasos los abandonos o fracasos clínicos en infecciones complejas.

Más preocupante es su eventual uso inapropiado o sobreuso, pues indudablemente resultará en una mayor resistencia bacteriana, que por cierto ya se ha comunicado en las experiencias clínicas detalladas en esta revisión, donde se verifica la aparición de cepas con susceptibilidad reducida o no susceptibles a tigeci- 
clina, a veces durante el curso del tratamiento con el mismo antimicrobiano ${ }^{16,18,19,21}$. Por lo mismo, debiera ser una tarea importante para los especialistas en enfermedades infecciosas y profesionales farmacéuticos cautelar el uso prudente de este nuevo antibacteriano, reservándolo fundamentalmente como una terapia de rescate para patógenos multi-resistentes, tales como SAMR, ERV, A. baumannii y otros bacilos gramnegativos productores de BLEE y/o carbapenemasas.

\section{Conclusiones}

Tigeciclina es un fármaco eficaz en el tratamiento de infecciones de piel y tejidos blandos complejos, infecciones intra-abdominales complicadas y neumonías adquiridas en la comunidad. Salvo los eventos adversos gastrointestinales, es un fármaco muy bien tolerado. $\mathrm{Su}$ amplio espectro antibacteriano, que incluye agentes altamente resistentes a otros antimicrobianos, tales como SAMR, ERV, Enterobacteriaceas productoras de BLEE y A. baumannii, lo hace una alternativa particularmente útil en el manejo de infecciones debi- das a estos patógenos. Sin embargo, la emergencia de resistencia como peligro potencial debe guiar su uso juicioso en el medio intrahospitalario.

\section{Resumen}

Se analiza, bajo la perspectiva de una revisión sistemática de la literatura científica, la experiencia clínica con tigeciclina en las indicaciones ya aprobadas por las entidades reguladoras y en aquellas indicaciones off label reportadas en la literatura médica reciente. Se revisa el perfil de seguridad y tolerabilidad en dichos ensayos clínicos. La información disponible permite avalar su eficacia en el manejo de de infecciones de piel y tejidos blandos complejas, infecciones intrabdominales complicadas y neumonías adquiridas en la comunidad. Se insinúa además su utilidad en el manejo de infecciones por patógenos con alto nivel de resistencia a antimicrobianos. Sin embargo, se requiere de mayor evidencia al respecto y de una muy sensata política de uso en el medio hospitalario.

\section{Referencias}

1.- Chopra I. Glycylcyclines: Third-generation tetracycline antibiotics. Curr Opin Pharmacol 2001; 1: 464-9.

2.- Postier R G, Green S L, Klein S R, Ellis-Grosse E J, Loh E; for the Tigecycline 200 Study Group. Results of a multicenter, randomized, open-label efficacy and safety study of two doses of tigecycline for complicated skin and skin-structure infections in hospitalized patients. Clin Ther 2004; 26: 704-14.

3.- Sacchidanand S, Penn R L, Embil J M, Campos M E, Curcio D, Ellis-Grosse E J. Efficacy and safety of tigecycline monotherapy compared with vancomycin plus aztreonam in patients with complicated skin and skin structure infections: Results from a phase 3 , randomized, double-blind trial. Int J Infect Dis 2005;9: 251-61.

4.- Breedt J, Teras J, Gardovskis J, Maritz F J, Vaasna R, Ross D P, for the Tigecycline 305 cSSSI Study Group. Safety and efficacy of tigecycline in treatment of skin and skin structure infections: Results of a double-blind phase 3 comparison study with vancomycinaztreonam. Antimicrob Agents Chemother 2005; 49: 4658-66.

5.- Oliva M E, Rekha A, Yellin A, Pasternak J, Campos M E, Rose G M, for the Tigecyclin 301 Study Group. A multicenter trial of the efficacy and safety of tigecycline versus imipenem/cilastatin in patients with complicated intraabdominal infections. BMC Infect Dis 2005; 5: 88.

6.- Dartois N, Gioud-Paquet M, Ellis- Grosase E J, Loh E, for the Tigecycline 306 Study Group. Tigecycline vs imipenem/cilastatin for treatment of complicated intrabdominal infections. In: Abstracts of the $44^{\text {th }}$ Interscience Conference on Antimicrobial Agents and Chemotherapy. Washington, DC: American Society for Microbiology; 2004. Abstract L-992C.

7.- Tanaseanu C, Bergallo C, Teglia O, Jasovich A, Oliva M E, Dukart G, et al, on behalf of the 308 and 313 Tigecycline Study Groups. Integrated results of 2 phase 3 studies comparing tigecycline and levofloxacin in community-acquired pneumonia. Diag Microbiol Infect Dis 2008; 61 (3): 329-38

8.- Dartois N, Dukart G, Cooper C A, Castaing N, Gandjini H on behalf of the 308 and 313 Study Groups. Efficacy of tigecycline compared with levofloxacin for treating Streptococcus pneumoniae bacteraemia in patients hospitalised with communityacquired pneumonia. 17 th ECCIMD 2007. Poster 848

9.- Disponible en http://www.clinicaltrials.gov/ $\mathrm{ct} /$ search?term=tigecycline \&submit=search (accedido: 129 febrero 2008).
10.- Ellis-Grosse E J, Babinchak T, Dartois N, Rose G, Loh E, et al, for the Tigecycline 300 cSSSI Study Group and the Tigecycline 305 cSSSI Study Group. The efficacy and safety of tigecycline in the treatment of skin and skin-structure infections: Results of 2 double-blind phase 3 comparison studies with vancomycin-aztreonam. Clin Infect Dis 2005; 41 (SuppI 5): S341-53.

11.- Babinchak T, Ellis-Grosse E, Dartois N, Rose G M, Loh E, et al, for the Tigecycline 301 Study Group and the Tigecycline 306 Study Group. The efficacy and safety of tigecycline for the treatment of complicated intra-abdominal infections: Analysis of pooled clinical trial data. Clin Infect Dis 2005; 41 (Suppl 5): S354-67.

12.- Muñoz-Price L S, Lolans K, Quinn J P. Four cases of invasive methicillin-resistant Staphylococcus aureus (MRSA) infections treated with tigecycline. Scand J Infect Dis 2006; 38: 1081-4.

13.- Denton M, Purcell N, Cunliffe H, Brownlee K, Peckham D, Conway S. Use of tigecycline to treat difficult respiratory pathogens in cystic fibrosis-early experiences. J Cystic Fibrosis 2007; 6 (Suppl 1): S15.

14.- Chemaly R, Hachem R, Hanmod S, Adachi J, Hogan H, Mulanovich V, et al. Tigecycline usage in cancer patients with refractory pneumonia: a report on 38 cases. A single- 
institution study. En: Program and Abstracts of the $17^{\text {th }}$ European Congress of Clinical Microbiology and Infectious Diseases; 31 March - 3 April 2007, Munich, Germany, Abstract P729.

15.- Schafer J J, Goff D A, Stevenson K B, Mangino J E. Early experience with tigecycline for ventilator-associated pneumonia and bacteremia caused by multidrug-resistant Acinetobacter baumannii. Pharmacotherapy 2007; 27: 980-7.

16.- Lewis J S, Echevarria K, Owens A, Jorgensen $\mathrm{J} \mathrm{H}$. Experience with the use of Tigecycline (TIG) in critically ill patients. 45 ${ }^{\text {th }}$ Annual Meeting of IDSA, 2007. Abstr 1077. Pag 245.

17.- Burgos J, Wehbeh, Mangini E, Figueroa D, Urban C, Mariano N, et al. Efficacy of tigecycline $(\mathrm{T})$ in infections due to multidrug resistant (MDR) Klebsiella pneumoniae. $45^{\text {th }}$ Annual Meeting of IDSA, 2007. Abstr 1092. Pag 248.

18.- Leclerc T, Cadé S, Pérez J P, Soler C, Debien B, Clapson P, et al. Treatment of a severe pulmonary infection due to multidrug-resistant Acinetobacter baumannii with tigecycline followed by emergence and spread of a tigecycline-resistant clone. En: Program and Abstracts of the $47^{\text {th }}$ Interscience Conference on Antimicrobial Agents and Chemotherapy [on CDROM].Washington, DC: American Society for Microbiology; 2007. Abstract K-391

19.- Muñoz-Price L S, Zembower T, Schreckenberger P C, Harton L, Mohapatra S, Baig M O, et al. Tigecycline $v s$ colistin for the treatment of multidrugresistant Acinetobacter baumannii blood stream infection. En: Program and Abstracts of the $48^{\text {th }}$ Interscience Conference on
Antimicrobial Agents and Chemotherapy [on CD-ROM]. Washington, DC: American Society for Microbiology; 2008. Abstract K389

20.- Swoboda S, Ober M, Hainer C, Lichtenstern C, Seiler C, Wendt C, et al. Tigecycline for the treatment of patients with severe sepsis or septic shock: a drug use evaluation in a surgical intensive care unit. J Antimicrob Chemother 2008; 61: 729-33.

21.- Paramythiotou E, Kontopidou F, Papadomichelakis E, Antoniadou A, Poulakou G, Armaganidis A, et al. Tigecycline in ICU Infections. En: Program and Abstracts of the $18^{\text {th }}$ European Congress of Clinical Microbiology and Infectious Diseases; April 19-22, 2008; Barcelona, España, Abstract 554

22.- Jenkins I. Linezolid- and vancomycinresistant Enterococcus faecium endocarditis: Successful treatment with tigecycline and daptomycin. J Hosp Med 2007; 2: 5; 343-4.

23.- Cobo J, Morosini M I, Pintado V, Tato M, Samaranch N, Baquero F, et al. Use of tigecycline for the treatment of prolonged bacteremia due to a multiresistant VIM-1 and SHV-12 $\beta$-lactamase-producing Klebsiella pneumoniae epidemic clone. Diag Microbiol Infect Dis 2008; 60: 319-22

24.- Antony S, Domínguez D C. Use of a novel antibiotic (tigecycline) in the treatment of peritoneal dialysis-associated MRSA peritonitis. Dialysis \& Transplantation 2008; 37: 30.

25.- Krueger W A, Volkhard A, Kempf J, Peiffer M, Nagele U, Unertl K E, et al. Treatment with tigecycline of recurrent urosepsis caused by extended-spectrum- $\beta$-lactamaseproducing Escherichia coli. J Clin Microbiol 2008; 817-20.

26.- Taccone F S, Rodríguez-Villalobos H,
De Backer D, De Moor D, Deviere J, Vincent J L, et al. Successful treatment of septic shock due to pan-resistant Acinetobacter baumannii using combined antimicrobial therapy including tigecycline. Eur J Clin Microbiol Infect Dis 2006; 25: 257-60.

27.- Saner F H, Heuer M, Rath P M, Gensicke J, Radtke A, Druhe N, et al. Successful salvage therapy with tigecycline after linezolid failure in a liver transplant recipient with MRSA pneumonia. Liver Transplantation 2006; 12: 1689-92.

28.- Daly M W, Riddle D J, Ledeboer N A, Dunne W M, Ritchie D J. Tigecycline for treatment of pneumonia and empyema caused by carbapenemase-producing Klebsiella pneumoniae. Pharmacotherapy 2007; 27: 1052-7.

29.- Stanzani M, Tumietto F, Giannini M B, Bianchi G, Nanetti A, Vianelli N, et al. Successful treatment of multi-resistant Pseudomonas aeruginosa osteomyelitis after allogeneic bone marrow transplantation with a combination of colistin and tigecycline. J Med Microbiol 2007; 56: 1692-5.

30.- Rolfe N E, Toney J F, Green M R, Sandin R L, Greene J N. Successful treatment of Mycobacterium fortuitum osteomyelitis after allogeneic bone marrow transplantation. Infect Dis Clin Pract 2007; 15: 339-40.

31.- Swoboda S, Hoppe-Tichy T, Geiss H K, Hainer C, Nguyen T H, Knaebel H P, et al Septic shock due to vancomycin-resistant enterococci infection. Tigecycline monotherapy. Anaesthesist 2007; 56: 169-74.

32.- Tygacil, información de prescripción. En http://www.wyeth.com/hcp/tygacil/ prescribing (accedido 28 febrero 2008). 